\title{
Mobilisation after a bilateral Transmuscular Quadratus Lumborum block A local quality survey
}

\author{
Katrine Tanggaard', Charlotte Bernhoff', Martin Vedel Nielsen', Jens Børglum'
}

'Department of Anaesthesiology \& Intensive Care Medicine, Zealand University Hospital, University of Copenhagen, Denmark

\section{Background}

An ultrasound-guided bilateral Transmuscular Quadratus Lumborum(TQL) block provides analgesia of both the somatic and visceral nerves in the abdomen due to the spread of injectate to the thoracic paravertebral space ${ }^{1}$. A cadaveric study has previously showed no spread of injectate to the lumbar plexus ${ }^{1}$.

One case of unilateral lower limb weakness has been described after a bilateral TQL block (anterior to posterior approach, supine positioning ${ }^{2}$.

Another research group has, without mentioning their block technique, described that $90 \%$ of their patients experience lower limb weakness when performing TQL blocks ${ }^{3}$. We wanted to survey the incidence of lower limb weakness when performing a preoperative bilateral TQL block at our department.

\section{Methods}

During two weeks of Spring 2019 is was registered whether the patients having a preoperative bilateral ultrasound-guided TQL blocks showed sign of lower limb motor weakness afterwards (ability to walk to the operating theatre). The Transmuscular Quardratus Lumborum (TQL) block is performed with the patient in a lateral decubitus position. A curved array transducer is placed in the posterior axillary line immediately above the iliac crest. Using an in-line posterior approach the local anaesthetic is injected in the interfascial plane between the quadratus lumborum and the psoas major muscles.

\section{Results}

Eight patients had a pre-operative bilateral TQL-block with $2 \times 30 \mathrm{~mL} 0.375 \%$ ropivacaine in our block room approximately 30 minutes prior to entering the operating theatre. All eight patients $(100 \%)$ were, after having a bilateral TQL block, able to walk independently from the holding area and into the operating theatre.

\section{Conclusion}

From our mini survey no patients showed sign of lower limb weakness after a pre-operative bilateral TQL-block. The TQL block is an advanced block and the previously described lower limb weakness might be due to an accidental lumbar plexus block.

\section{References}

1) Dam M, Moriggl B, Hansen CK, Hoermann R, Bendtsen TF, Børglum J. The Pathway of Injectate Spread With the Transmuscular Quadratus Lumborum Block: A Cadaver Study. Anesth Analg. 2017 Mar 8. PMID: 28277325.

2) Wikner M. Unexpected motor weakness following quadratus lumborum block for gynaecological laparoscopy. Anaesthesia. 2017 Feb;72(2):230-232.

3) Ueshima $\mathrm{H}$, Hiroshi O. Incidence of lowerextremity muscle weakness after quadratus lumborum block. J Clin Anesth. 2018 Feb; 44:104. PMID: 29175750.

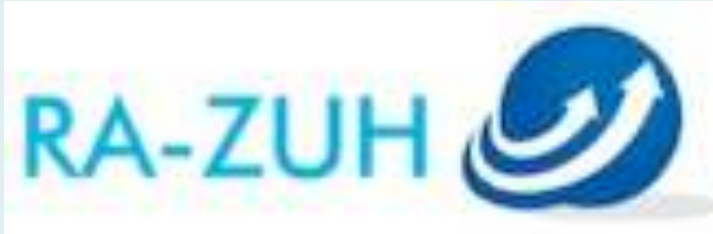

